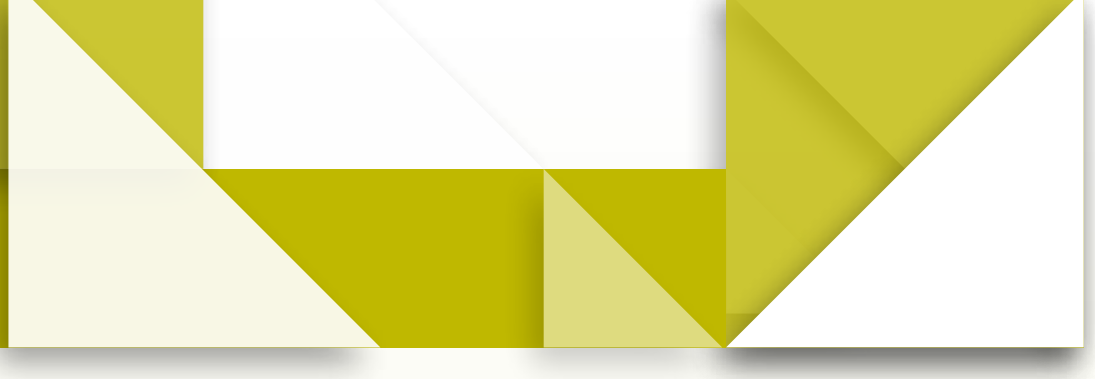

\title{
Desarrollo del pensamiento crítico y reflexivo a partir de la enseñanza del sonido
}

\section{- Development of Critical and Reflective Thinking Based on Teaching of Sound \\ - Desenvolvimento do pensamento crítico e reflexivo a partir do ensino do som}

\section{Resumen}

Partiendo de la perspectiva dimensional de la didáctica de la física, que contempla el dominio del profesor en lo disciplinar, lo sociocultural y lo interaccional en la clase, definimos como problema de estudio al desarrollo de habilidades de pensamiento crítico y reflexivo por medio de la clase de física. Esta fue una investigación-acción con estudiantes de grado once y su docente, en un colegio público de Bogotá. Para ello, se diseñó una estrategia metodológica de enseñanza del sonido en fases, con el objetivo de estimular cinco habilidades para el pensamiento crítico: análisis, interpretación, inferencia, evaluación y autorregulación. Se desarrollaron tres ciclos de análisis orientando a los estudiantes en niveles de participación, desarrollo de lenguaje, formulación de argumentos, y autorreconocimiento de procesos de aprendizaje. Abordamos conceptos referentes a cualidades del sonido desde el análisis de los efectos de la música y la clasificación de instrumentos, hasta conceptualizar características de las ondas sonoras. Encontramos que es posible desarrollar el pensamiento crítico reflexivo en el aprendizaje de conceptos como tono, timbre e intensidad del sonido, ya que tienen mucho que ver con las experiencias de vida de los estudiantes. Observamos, también, que tanto los estudiantes como la docente, mejoraron su disposición para el debate, la expresión de diversidad de ideas y la argumentación, con el fin de consolidar sus explicaciones y afinar sus organizaciones conceptuales. Hubo reformulaciones sobre sus imaginarios acerca del significado de enseñar y aprender física, más allá de la transmisión de contenidos.

Palabras clave

didáctica de la física; teoría de la educación; enseñanza del sonido

\section{Yenny Lucila Fonseca* Olga Lucía Castiblanco**}

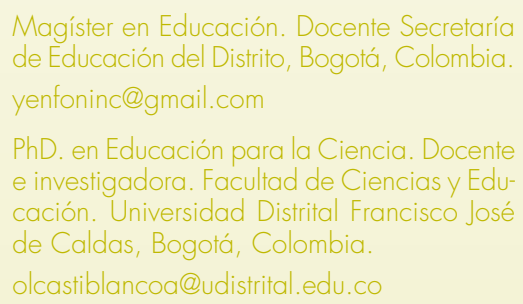

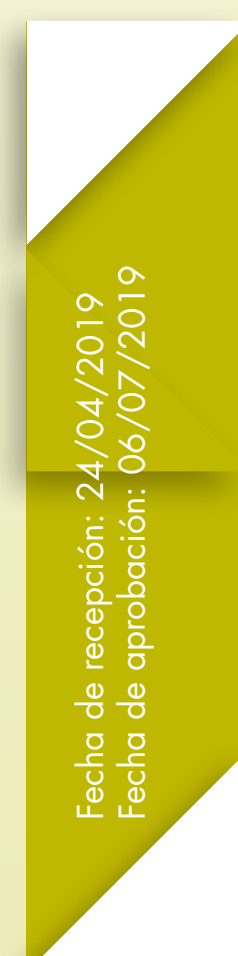




\section{Abstract}

Based on a dimensional perspective of the didactics of physics, that includes teacher's domain in disciplinary, socio-cultural and interactional aspects of the class, we define as study problem, the development of critical and reflexive thinking skills through the physics class. This was an action-research with students in high school, in Bogotá. To this end, it was necessary to design a methodological strategy to teach the concept of sound in phases, looking for stimulating five skills to critical thinking: analysis, interpretation, inference, evaluation and self-regulation. Three cycles of analysis were developed, orienting students in levels of participation, language development, formulation of arguments, and self-recognition of their learning processes. We approach concepts related to sound qualities starting from the analysis of the effects of music and the classification of instruments, until conceptualizing the characteristics of sound waves. We found that it is possible to develop reflexive critical thinking in the learning of concepts such as pitch, timbre and intensity of sound since they have a lot to do with the students' life experiences. We also observed that both, the students and the teacher, improved their disposition for the debate, expression of diverse ideas and argumentation. All the above, to consolidate their explanations and refine their conceptual organizations. There were reformulations about imaginaries in relation to the significance of teaching and learning physics beyond the transmission of content.

Keywords

didactics of physics; educational theory; sound teaching

\section{Resumo}

Partindo da perspectiva dimensional da didática da física, que exige o domínio do professor no disciplinar, sociocultural e interacional na sala de aula, definimos como problema de estudo o desenvolvimento de habilidades de pensamento crítico e reflexivo através do ensino de física. Esta foi uma pesquisa ação com estudantes de ensino meio em Bogotá. Para isso, planejamos uma estratégia metodológica de ensino do som em fases, objetivando estimular cinco habilidades de pensamento crítico: análise, interpretação, inferência, avaliação e auto regulação. Desenvolvemos três ciclos de análise, orientando aos alunos em níveis de participação, acréscimo de sua linguagem, formulação de argumentos e auto reconhecimento de processos de aprendizagem. Em termos da física, abordamos conceitos relacionados a qualidades do som, partindo da análise dos efeitos da música e a classificação de instrumentos, até chegar na conceptualização das características das ondas sonoras. Encontramos que é possível desenvolver o pensamento crítico e reflexivo na aprendizagem de conceitos como tom, timbre e intensidade do som, porque tem muito a ver com as experiências de vida dos estudantes. Observamos também, que tanto os alunos quanto a professora, melhoraram sua disposição para o debate, a expressão de diversidade de ideias e a argumentação, a fim de consolidar suas explicações e aprimorar suas organizações conceituais. Houve reformulações sobre seus imaginários do que significa ensinar e aprender física para além da transmissão de conteúdos.

Palavras chave

didática da física; teoria da educação; ensino do som 


\section{Introducción}

En este trabajo, se asume el pensamiento crítico como un pensamiento razonado y reflexivo, orientado a la toma de decisiones sobre qué hacer, en qué creer, como dudar, como proponer nuevas ideas, aspectos que resultan importantes en la educación de los sujetos y, particularmente, en la enseñanza de las ciencias. De acuerdo con Sanmartí (2002), se asume que todas las personas deben ser educadas en ciencias teniendo en cuenta la formación del pensamiento crítico, de modo que mejoren el proceso de toma de decisiones, por ejemplo, respecto a problemáticas de tipo ambiental, del cuidado y la salud, del uso de la ciencia y la tecnología.

Así, la enseñanza de las ciencias debe ir más allá de la ilustración en teorías, leyes y conceptos, y ofrecer, tanto a los estudiantes como al docente, oportunidades de comprensión y explicación de lo que ocurre a su alrededor. Sin embargo, paradójicamente, muchas veces la enseñanza de la física suele educar para la aprehensión de verdades absolutas aisladas de las experiencias de vida y sin posibilidad de análisis y reflexión. Por ello, se asume el reto de pensar sobre cómo educar a los estudiantes para la crítica reflexiva por medio de procesos de enseñanza y aprendizaje de la física, en donde el docente también crezca en el mismo sentido. Es decir, lograr la emancipación individual y colectiva, entendida como la capacidad de reflexionar sobre las verdades absolutas para crear argumentos propios.

\section{Marco de referencia}

\section{El pensamiento crítico en la educación}

En la perspectiva de Gardner (2004), los profesores tienen la misión de cambiar la mente de los estudiantes, y mientras la escuela no asuma este compromiso, no se estará educando adecuadamente para la sociedad actual y futura, ya que desarrollar las formas de pensar implica la formación del pensamiento crítico y reflexivo sobre su vida misma. Pero son diversos los aspectos que se deben transformar en el aula.

Por ejemplo, el docente debe aprender a re-pensar los contenidos que enseña, y tomar conciencia del dominio y pertinencia de estos, así como del lenguaje con el que habla de la ciencia y se comunica con los estudiantes. Por su parte, los estudiantes deben ser educados para la autonomía, lo que requiere formarlos para la participación activa, en donde se sientan capaces de opinar, cuestionar, cooperar en el aula y desarrollar su propio discurso sobre los temas tratados en clase. A su vez, la institución educativa debe propiciar ambientes en donde docentes y estudiantes desarrollen actividades no protocolizadas por agentes externos y se puedan reconocer como potenciales productores de conocimiento en diferentes niveles y áreas.

Lo anterior, de acuerdo con Castiblanco y Vizcaíno (2006), requiere comprender el significado de desarrollar procesos metacognitivos en la clase, así como de autorreconocimiento de cada ser humano para que se comprenda a sí mismo y a los demás. También implica la resignificación del sentido de la enseñanza que, para el caso de las ciencias naturales, no deberá estar centrado en el contenido, sino en el sujeto, y en las posibilidades de crecimiento humano y social que ofrece la clase, sabiendo que, particularmente la física, posibilita oportunidades de desarrollo del pensamiento para la argumentación, el análisis, la comprensión del mundo, entre otras.

En esta misma línea, autores como Giroux (1988) defienden que el acto de aprender es un ejercicio de liberación y de humanización. Sin embargo, se requiere de profesores reflexivos que, para lograrlo, se asuman como intelectuales y productores de conocimiento, que puedan crear metodologías de enseñanza contextualizadas, que articulen la diversidad de saberes y sean responsables social y políticamente. La enseñanza, por tanto, debe darse en un ambiente en donde los estudiantes y el

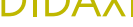


profesor reflexionen sobre lo que acontece en el aula y a partir de allí surja la construcción de nuevos conocimientos y procesos de toma de decisiones.

\section{Aproximaciones al significado del pensamiento crítico reflexivo}

El pensamiento crítico ha sido ampliamente documentado y analizado en diversos estudios. De acuerdo con Saiz (2002) puede entenderse como un proceso elaborado de pensamiento racional, autorregulado, consciente y con un propósito determinado, compuesto de habilidades, disposiciones y motivaciones para obtener mejores resultados en la búsqueda de conocimiento, solución de problemas y toma de decisiones. El autor también se refiere a la contradicción existente entre el aumento de la cantidad de información a la que están expuestas las personas y los bajos niveles de razonamiento, lo cual crea la necesidad de desarrollar habilidades para el análisis con el fin de ubicarse mejor en la sociedad como sujeto partícipe de lo que allí ocurre.

Lo anterior concuerda con la perspectiva de León (2014) quien define pensamiento crítico como los procesos, estrategias y representaciones mentales que la gente usa para resolver problemas, tomar decisiones y aprender nuevos conceptos, a fin de emitir juicios con ciertos grados de validez. Igualmente, permite que los sujetos autoexaminen su coherencia, fundamentación, intereses y valores. Por su parte, para Ennis (2013) existen dos condiciones que se deben dar en el sujeto para que pueda emprender el desarrollo del pensamiento crítico, una es la disposición de la persona para trabajar sobre su propia manera de pensar y otra es la capacidad cognitiva necesaria para pensar de modo crítico.

Hay algunas habilidades de pensamiento claramente definidas que se pueden desarrollar por medio de procesos de enseñanza y aprendizaje de las ciencias naturales; entre ellas: la capacidad de comprender relaciones de causalidad, valorar suposiciones, defender un punto de vista, construir argumentaciones, elaborar conclusiones, sopesar grados de incertidumbre, utilizar diversas estrategias para resolver problemas.

Por tanto, el diseño de tales estrategias debe estar basado en ejercicios de tipo metacognitivo, dinámicas de interacción que posibiliten otros modos de relación entre los participantes de la clase y acercamientos al contenido disciplinar desde acciones diferentes al mero análisis teórico de los modelos matemáticos explicativos, como lo desarrollan Castiblanco y Nardi (2017), en su perspectiva dimensional de la didáctica de la física.

Con el fin de orientar el diseño metodológico de los ciclos de análisis en esta investigación, tomamos como referente a Facione (1992) cuando delimita algunas fases de desarrollo del pensamiento crítico reflexivo en el aula, entendiendo que el objetivo es lograr que los estudiantes y la docente titular desarrollen destrezas de interpretación, análisis, evaluación, inferencia, explicación y autorregulación. Para evidenciar la presencia de dichas habilidades nos apoyamos en Torres y Solbes (2014), quienes caracterizan el desarrollo del pensamiento crítico como aquel que "promueve la autonomía de opinión, el análisis y la reflexión de situaciones, la formulación de juicios fundamentados, el cuestionamiento de la información" (p. 58). 
A continuación, presentamos las definiciones que decantamos para cada una de las habilidades de pensamiento y que tomamos como base para este trabajo:

- Análisis. Identificar las relaciones causa/ efecto obvias o implícitas en las diferentes ideas expuestas.

- Inferencia. Extraer información pertinente para decidir sobre la aceptabilidad y consecuencias de una alternativa ideoló-

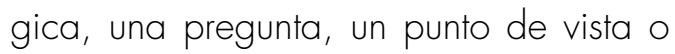
una teoría.

- Interpretación. Describir experiencias, situaciones, creencias, eventos, con significados comprensibles en términos de categorizaciones, distinciones o marcos de referencia.

- Evaluación. Valoración de la credibilidad de las representaciones y relaciones que dan cuenta del desarrollo de un proceso.

- Autorregulación. Monitorear en forma consciente las actividades cognitivas, reflexionar sobre los valores, motivaciones, actitudes e intereses para determinar si se ha sido ecuánime, objetivo y justo. Tener la capacidad de diseñar procedimientos para enmendar errores.

En cada ciclo de trabajo estimulamos a los estudiantes para el desarrollo del análisis, la inferencia y la interpretación, asumiendo la evaluación y la autorregulación como transversales a todo el proceso.

\section{Metodología}

Esta es una investigación cualitativa que se fundamenta en la perspectiva de Flick (2009), quien la caracteriza como un camino para comprender, describir y explicar los fenómenos sociales y educativos desde diferentes perspectivas. Es de tipo investigación-acción que, según Elliot (1991), ofrece la posibilidad de consolidar un problema asociado al propio ejercicio docente y su solución con la cooperación de los estudiantes.

La problemática se configuró a partir de las reflexiones iniciales de la docente titular acerca de la necesidad de que sus estudiantes fuesen más participativos y encontraran mayor sentido a lo aprendido en clase. Por ello, se asumió el desarrollo del pensamiento crítico como la teoría que le daría sentido al cambio deseado. Así, los ciclos de investigación se basaron en la estimulación de tres habilidades de pensamiento, el análisis, la interpretación y la inferencia como fases consecutivas, sin querer decir que no existiera presencia de una en la fase de estímulo de otra, sino enfocando actividades especiales para estudiar el desarrollo de cada una.

Luego de acordar que el desarrollo del pensamiento crítico y reflexivo en los estudiantes no depende de un contenido específico de la física, sino de los modos de proceder en el aula, optamos por tratar el tema que curricularmente debería continuar de acuerdo con el currículo, que para el caso fue el concepto de onda sonora. El grupo de trabajo se conformó por la docente titular y 30 estudiantes de grado undécimo de un colegio público de Bogotá.

Cada ciclo debería tener una característica distintiva en las formas de interacción en el aula y los modos alternativos de estudiar el sonido. Con este fin, nos fundamentamos en la perspectiva dimensional de la didáctica de la física de Castiblanco y Nardi (2017) y Nardi y Castiblanco (2014), quienes señalan tres dimensiones en las que el profesor debe trabajar para re-pensar su ejercicio profesional.

La primera es analizar el nivel de dominio que tiene sobre el contenido a enseñar, no solo en el manejo de ecuaciones sino en la apropiación del lenguaje, la comprensión de la epistemología, la evolución del concepto a lo largo de la historia y los principios filosóficos que le sustentan, para que pueda determinar lo más apropiado a ser enseñado. La segunda es la comprensión del contexto identificando sus características, lo cual le permite establecer el significado y el sentido de enseñar una 
temática, no solo desde la mera aprehensión de nuevo conocimiento, sino principalmente sobre el aporte al desarrollo de la persona, como sujeto pensante y activo, así como sujeto social que aprende junto con otros. La tercera es el análisis de las formas posibles de interacción entre los participantes de la clase, es decir, el rol que puede desempeñar el docente y el estudiante, por ejemplo, en un debate, en una experiencia de laboratorio, en la resolución de un problema, o en las reflexiones.

Una vez que la profesora hizo este ejercicio de análisis, es decir, estudió lo esencial de las ondas sonoras, caracterizó su contexto y reconoció diferentes posibilidades de acción en el aula, se diseñaron las clases descritas a continuación. El instrumento de toma de datos fue una rejilla con indicadores de observación que la docente diligenció a partir de las grabaciones de audio de todas las sesiones de clase, los materiales escritos por los estudiantes como resultado de las diferentes actividades y los relatos de cada clase hechos por la docente. Esta rejilla fue diseñada previamente, atendiendo principalmente a la búsqueda de evidencias de la ocurrencia de procesos de análisis, inferencia, interpretación, evaluación y autorregulación.

\section{Resultados}

Presentamos los resultados de cada ciclo haciendo énfasis en la búsqueda de evidencias del desarrollo de la habilidad de análisis en el primer ciclo, pues esta habilidad fue la estimulada principalmente. En el segundo ciclo se estudió la habilidad de inferencia y en el tercer ciclo, la habilidad de la interpretación. Presentamos para cada una de las actividades desarrolladas, la metodología de interacción en el aula, así como los objetivos y la descripción de los recursos de apoyo utilizados.

\section{Estimulando la habilidad para analizar}

Iniciamos aplicando un cuestionario para indagar sobre sus concepciones acerca del pensamiento crítico y entablar un diálogo. Encontramos que lo definen, proponen cómo desarrollarlo, con qué tipo de actividades, cómo evaluarlo, y solicitaron tener más participación y discusión de los temas. En la tabla 1, sintetizamos las expresiones más frecuentes de los estudiantes.

En esta tabla observamos que los estudiantes asociaron el pensamiento crítico y reflexivo con la posibilidad de analizar, solucionar problemas y desarrollar ideas que les permitiesen dialogar con otros, ya sea para estar de acverdo o para debatir. En general, entienden que desarrollar el pensamiento se refiere a una actividad humana, tener ideas en la mente de algo, razonar, resolver problemas y analizar. Algunos de ellos dicen puntualmente,

E 1: Yo creo que pensar es tener varias ideas sobre un tema específico.

E2: Pensar es tener ideas, dudas, seguridad, ocupar la mente en situaciones.

E3: Pensar es la habilidad de poder interpretar una situación, problema u operación en el cual se le puede encontrar una múltiple respuesta... 
Tabla 1. Aspectos sobre el pensamiento crítico reflexivo, indicados por los estudiantes en el cuestionario inicial

\begin{tabular}{|c|c|c|c|c|}
\hline $\begin{array}{c}\text { Definición de } \\
\text { pensamiento crítico }\end{array}$ & $\begin{array}{l}\text { Sobre cómo } \\
\text { desarrollarlo }\end{array}$ & $\begin{array}{l}\text { Tipo de actividades para } \\
\text { favorecer su desarrollo }\end{array}$ & $\begin{array}{l}\text { Evaluación del } \\
\text { proceso }\end{array}$ & $\begin{array}{l}\text { Aspectos a modificar } \\
\text { en la clase }\end{array}$ \\
\hline $\begin{array}{l}\text { - Razonar sobre } \\
\text { algo. } \\
\text { - Encontrar respuestas } \\
\text { o solucionar } \\
\text { problemas. } \\
\text { - Tener ideas en } \\
\text { la mente. } \\
\text { - Sirve para resolver } \\
\text { problemas, en este } \\
\text { caso de física. }\end{array}$ & $\begin{array}{l}\text { - Analizar. } \\
\text { - Debatir. } \\
\text { - Estar de acuerdo } \\
\text { o no con algo } \\
\text { o alguien. } \\
\text { - Tener argumentos } \\
\text { para estar a favor } \\
\text { o en contra de una } \\
\text { idea u opinión. }\end{array}$ & $\begin{array}{l}\text { - Actividades al } \\
\text { aire libre, lúdicas } \\
\text { - J dinámicas. } \\
\text { - Juegos y experimentos. } \\
\text { - } \text { memividades de } \\
\text { lógico-mación y } \\
\text { - Diferentes estraticas. } \\
\text { para aprender. }\end{array}$ & $\begin{array}{l}\text { - Puntualidad y } \\
\text { responsabilidad. } \\
\text { - Cumplimiento } \\
\text { en tareas y } \\
\text { actividades. } \\
\text { - Esfuerzo hecho. }\end{array}$ & $\begin{array}{l}\text { - Las explicaciones, dado } \\
\text { que a veces son muy } \\
\text { rápidas o confusas. } \\
\text { - Valores agregados a } \\
\text { la clase, que llamen } \\
\text { la atención ya que a } \\
\text { veces los estudiantes } \\
\text { se distraen. } \\
\text { - Teoría y práctica } \\
\text { para hacer más } \\
\text { comprensibles los } \\
\text { temas, porque a veces } \\
\text { son muy difíciles. }\end{array}$ \\
\hline
\end{tabular}

Fuente: elaboración propia.

Aclaramos que usamos los códigos con la letra E para representar a un estudiante, seguida de un número que diferencia un estudiante de otro. Así, $E_{1}, E_{2}$ y $E_{3}$ son tres estudiantes que participaron.

Respecto a las habilidades de pensamiento requeridas, ellos indican principalmente la de resolver problemas de física. Igualmente, reconocieron que una forma de lograrlo es por medio del debate, el análisis y de actividades que posibiliten mayor interacción entre ellos, uniendo la teoría y la práctica, lo cual se distancia de la tradicional perspectiva de pensar en resolver problemas de lápiz y papel. Cuando indagamos por los modos en que se debería evaluar un proceso basado en el desarrollo de pensamiento crítico, mencionan acciones como el compromiso, la responsabilidad, la puntualidad y el esfuerzo realizado. Este es un indicador de que ellos conciben modos alternativos a la evaluación tradicional.

En general, resaltaron que debía mejorarse el tipo de explicaciones que la docente ofrece pues, según ellos, son confusos, difíciles e incompletos, sin dejar de reconocer que les falta más compromiso, lo cual da indicios de un ejercicio de reflexión. Este primer resultado indicaba un inconformismo por parte de los estudiantes acerca de cómo se desarrollaba la clase de física que a su vez hizo ver a la docente la necesidad de un cambio urgente, un rompimiento con lo que se venía haciendo. Así que se implementan las siguientes actividades.

\section{Aplicación de encuesta para estimular el análisis}

Objetivo: fomentar el debate a partir de la socialización de ideas por parte de los estudiantes, sobre un fenómeno rutinario que despierte su interés.

Metodología de interacción en el aula: desarrollar trabajo individual en dos momentos y posteriormente trabajo colaborativo para consolidar respuestas e intercambiar ideas.

Actividad: responder por escrito la pregunta ¿qué tipo de música escucha?, y ¿̇por qué?, para luego socializar sus respuestas con los compañeros. Responder la siguiente encuesta sobre percepciones y concepciones del sonido (tomada de Castiblanco, Vizcaíno e lachel, 2010).

1. El sordo no puede oír, porque:

a. Sus orejas están incompletas.

b. No siente vibraciones.

c. El cerebro no interpreta los sonidos.

d. Otro. ¿Cual? 
2. ¿Qué produce el sonido en las personas?
a. Sensaciones.
b. Comunicaciones.
c. Identidad.
d. Otro. ¿Cual?

3. Las preferencias musicales que tiene, dependen de:

a. Los instrumentos con los que se produce la música.

b. Los intérpretes musicales.

c. El ritmo musical.

d. Otro. ¿Cual?

4. El momento que elige para escuchar música depende de:

a. Su estado de ánimo.

b. Su tiempo libre.

c. Sus costumbres

d. Otro. ¿Cual?

Los estudiantes respondieron individualmente, luego compartieron en pequeños grupos y finalmente socializaron con la clase, mientras la docente escuchaba e iba generando un diálogo reflexivo para propiciar el debate.

En las respuestas dadas se notó el tipo de preferencias por diversos ritmos musicales, principalmente rap, salsa, vallenato y rock. Los estudiantes manifestaron escuchar música la mayor parte del tiempo e inclusive no poder hacer ciertas cosas sin música, por ejemplo, hacer las tareas. Hicieron conjeturas sobre los efectos que tiene escuchar una música u otra, por ejemplo, en términos de estados de ánimo o de momentos para compartir.

Al aplicar la encuesta buscábamos fomentar el análisis entendido como la habilidad para conectar causas y efectos, lo cual surgió al hablar sobre la pregunta de ¿por qué no escuchan los sordos? Ya que la mayoría buscaron causas que les resultaran comprensibles o evidenciables.

Algunos defendían que una persona no oye porque el cerebro no interpreta los sonidos, es decir, que identifican una función del cerebro en el proceso de audición y no asignan al órgano del oído toda la función auditiva. Otros lo asociaron con la posibilidad de recibir vibraciones, pero sin tener claro el funcionamiento del oído, aun así, anticipando que algo debe vibrar para que ocurra la audición. Varias opciones ubican la causa en malformaciones internas, defectos de nacimiento, enfermedad o a que el oído está incompleto o dañado y reflexionan sobre la importancia de tener oídos para conectarse con el mundo.

Solo tres estudiantes no expusieron sus ideas, pero escucharon las de sus compañeros, lo cual seguramente les hizo reflexionar. Afirmamos esto debido a que, en las siguientes actividades, estos aumentaron su participación. Frente a la pregunta sobre los efectos del sonido en las personas, más de la mitad de ellos respondió que el sonido produce sensaciones como alegría, tristeza, emoción. Dos respondieron que el sonido le permite a una persona expresarse y comunicarse. 
A partir de allí se generaron diálogos, lo cual entendimos como un indicador de mejora en la disposición para el desarrollo de su pensamiento, ya que se sintieron más motivados a participar, a cuestionar a sus compañeros y a la profesora. Por su parte la docente, que representaremos con la letra $D$, se mostraba sorprendida de ver a algunos estudiantes participando, especialmente aquellos que ella consideraba apáticos, según los relatos que iba haciendo de la clase. Se encontraron expresiones como,

D: Quedé sorprendida de ver aquella estudiante $\mathrm{E}_{5}$ que nunca la había visto participar en la clase, pero además noté que es bien crítica y cuestiona todo. [...] es curioso que vaya perdiendo el año si se nota que es muy inteligente.

Es de resaltar que fuimos transitando de hablar de música a hablar del fenómeno del sonido. Las explicaciones surgieron de los conocimientos y experiencias que tenían los estudiantes con respecto al acto de oír o escuchar. Veamos algunas de las conclusiones obtenidas en los diferentes grupos, cuando se hizo el análisis y la socialización de las diferentes respuestas a cada una de las preguntas de la encuesta. Cada grupo está representado con la letra $G$ seguida de un número y estaba conformado por tres estudiantes.

G1: Cada sonido se constituye por vibraciones de diferente frecuencia, entonces el tímpano puede no captar algunas...

G3: Las ondas tienen energía, la energía es la que transmite las vibraciones... en sí el sonido sería energía transmitida por vibraciones [...]. Los sonidos se detectan a través de vibraciones...

G4: Por eso es que el ciego tiene que tocar todo... por las vibraciones...

G5: Por eso descarta uno de una vez la opción a) [...] que las orejas están incompletas... porque es interno.
Nótese que los estudiantes pudieron expresar sus puntos de vista con respecto a situaciones vivenciadas o de las que tienen conocimiento sobre el funcionamiento del oído y su relación con el sonido. También, escucharon a sus compañeros y compararon las diferentes opiniones que además fueron enriquecidas entre sí por la variedad de situaciones en donde ellos identifican la presencia de ondas sonoras.

Al analizar los conceptos expresados por los estudiantes encontramos que usan términos cercanos a las definiciones dadas por la ciencia establecida, como afirmar que el sonido está constituido por vibraciones que llevan consigo energía, lo cual fue una oportunidad para continuar profundizando en el tema. Las actividades permitieron que los estudiantes pudieran conectar el hecho de que una persona no pueda oír con el fenómeno de producción del sonido. Es decir, toman elementos de su experiencia de vida, para apoyarse en la construcción de conceptos de la física.

\section{Estimulando la habilidad de interpretación}

Buscamos dar continuidad a las inquietudes generadas anteriormente. La docente tomó especial cuidado con mejorar la orientación del debate para hacerlos reflexionar un poco más sobre sus ideas y también se dispuso a deshacer la organización tradicional del curso en filas para trabajar en grupos.

\section{Diálogo sobre vivencias y experimentación}

Objetivo: caracterizar las formas como se produce y percibe el sonido a partir de la experimentación y observación.

Metodología de interacción en el aula: trabajo individual para resolver la pregunta y socialización. Posteriormente, trabajo en grupo para el desarrollo experimental.

Actividad: responder la pregunta: ¿Qué instrumentos musicales conoce? Luego, clasificar el listado de instrumentos mencionados para encontrar aspectos comunes. Por último, 
observar el experimento del movimiento de la llama de una vela causado por el sonido de percusión en diferentes frecuencias e intensidades, como se observa en el montaje de las figuras 1 y 2.
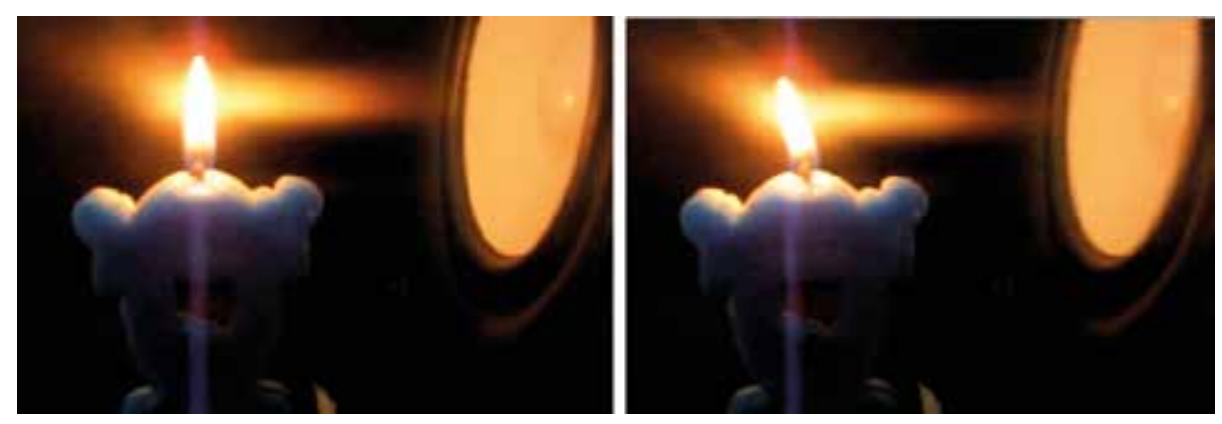

Figura 1. Movimiento de la llama de una vela causado por ondas sonoras percutidas

Fuente: Castiblanco, Vizcaíno e lachel (2010).

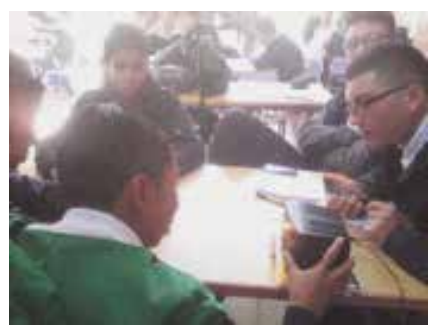

Figura 2. Estudiantes trabajando en grupo en torno a la experiencia de hacer mover la llama de una vela como efecto de la producción de sonidos con diferente frecuencia e intensidad.

Fuente: elaboración propia.

En la actividad de clasificación de los instrumentos musicales, se les entregó material con imágenes de diversos instrumentos. Surgieron categorías en donde incluyen aquellos que conocen y otros que solo han escuchado que existen. Al pedirles que clasificaran los instrumentos según sus características, llegaron a tres categorías: los instrumentos de cuerda que se pulsan, los de viento que se soplan y los de percusión que se golpean o mueven para que suenen.

Este resultado indica una introducción de los estudiantes hacia el estudio del sonido como fenómeno físico, ya que encuentran modos de hablar del sonido desde la manera en que se produce el sonido, y empiezan a hacer algunas inferencias al hablar del tipo de sonidos que producen las cuerdas o las membranas o los instrumentos con agujeros. Evidenciamos un enriquecimiento de su lenguaje y un esfuerzo por teorizar a partir de sus argumentaciones.

G 1: El instrumento de percusión [...] puede ser un bombo, no un bombo sino un mazo en el momento del contacto con el instrumento suelta un sonido, en cambio los de viento es el momento del aire, pues sí, los de viento en el momento del contacto con el aire se forma el sonido y los de guitarras con cuerdas...

G3: Pues nosotros cogimos viento, percusión, cuerda, de tocar y otro porque nosotros no sabíamos, de viento el que lo utiliza para que pueda sonar, no tocarlo como tal sino para que... expulse un sonido se hace presión con la boca...

G4: Pues también lo mismo cuerda, percusión y aire... los que nosotros pusimos en cuerda como los más conocidos guitarra, piano, violín, arpa... bueno 
y entre otros... que... la manipulación de los instrumentos es con los dedos... los de percusión los cogimos como por géneros musicales, la música como tal, por ejemplo, están las bases que se conocen percusión y le dan el ritmo a la música... y... los de viento que necesitan aire para funcionar.

Frente al experimento de la vela, los estudiantes observaron el movimiento de la llama y buscaron explicaciones. Allí utilizaron el término de onda y lo asociaron a alguna acción que ocurre entre la fuente del sonido y la vela. Algunos de los grupos respondieron así:

G1: Pues nosotros observamos que las ondas hicieron que como que el fuego de la vela... como que temblara...

G2: La vela nunca se quedó quieta, solo hasta que usted paro la música.

Esto evidencia que aun cuando la percusión se produce a una cierta distancia de la llama y que a simple vista no se observa que algo material se mueva desde la membrana en donde se produce la percusión hasta la llama, ellos sí asocian a algún tipo de movimiento en el ambiente que conecta la percusión y la llama de la vela.

G3: Si cuando el sonido iba pasando se incrementaba como la frecuencia y eso hacía que la vela se moviera más.

G4: La escala es que va de bajo a alto, que las ondas de sonido cada vez van más duro y la vela iba haciendo más ondas, el sonido producía ondas y el fuego de la vela se movía.

La docente utilizó estas declaraciones para orientar el debate sobre las características de las ondas sonoras. Aprovechó que los estudiantes hablaron de tonos en el sonido, denominados como escala, que perciben como un aumento o disminución de algo que se escucha, como ir de sonidos graves a sonidos agudos.

Algunos grupos inclusive acuñaron el término de frecuencia asociado al mayor o menor movimiento de la llama. Finalmente, un grupo afirmó que el sonido produce ondas que pueden ser más o menos fuertes o que pueden viajar en mayor o menor cantidad, lo cual da inicio a una discusión sobre si mayor y menor volumen del sonido está relacionado con mayor y menor frecuencia.

En este debate aparecieron argumentos que les aclararon que se puede tener una nota aguda y subirle y bajarle el volumen, sin que se afecte la frecuencia, lo cual quiere decir que la frecuencia no depende del volumen sino de lo agudo o grave. En medio del debate aparece la palabra decibeles que lleva a que todos se pregunten sobre su significado, lo cual es una oportunidad para que la docente desarrolle la idea sobre la intensidad de las ondas sonoras asociada al volumen.

Lo anterior propició el debate con los estudiantes y facilitó la construcción colectiva de una explicación basada en las características del sonido, como tono, timbre e intensidad. Estas son algunas de las expresiones que surgieron en medio del debate:

E7: Cuando el sonido llegó como más agudo, ya la llama no se movía y como el sonido más bajo sonaba duro y bajaba la frecuencia, por eso la llama se movía...

E8: Por eso... eso es lo que está haciendo que se mueva la vela, las ondas...

E9: Es que cuando el sonido está más grave, el bafle vibra y eso es lo que hace que la vela se mueva...

E10: Para mí... son las ondas las que producen la vibración.

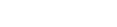

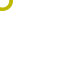


construido significados comprensibles para ellos aun cuando, por el momento, no coincidan exactamente con las explicaciones ofrecidas por la ciencia.

La interpretación se hizo evidente al pasar de hablar de música, a instrumentos musicales y luego del sonido. Los estudiantes hicieron abstracciones y lanzaron hipótesis sobre un experimento no identificable a simple vista, donde se requiere que el observador teorice en alguna medida, tal como se observa en las siguientes declaraciones:

E 1: Por eso, porque al momento que la canción iba progresando, iba subiendo el tono, entonces era más agudo y entonces, qué pasaba, que las ondas eran más cortas y no tenían espacio para vibrar, entonces por eso la vela no se movía...

E2: Agudo es cuando se producen más... vibraciones, mayor cantidad de ondas, o sea son más unidas y grave son más separadas...

E3: Por eso no hubo momento en que el sonido fuera grave, siempre aumentó, sí... o sea, aumenta el número de vibraciones, entonces el sonido va a ser más agudo...

E5: Entre más fuerte era el sonido, menos vibración tenía la vela, pero llego un momento en que la vela empezó a cargarse...

En la explicación se pretendía dar cuenta de ese conocimiento que los estudiantes construyeron a través de la observación y experimentación, las ideas que presentaron se acercaban cada vez más al conocimiento científico, aunque expresado en su lenguaje. Se evidenció la autorregulación cuando reflexionaron sobre sus propios errores conceptuales o de la falta de argumentación consistente, y fueron capaces de corregirse y aceptar que se equivocaron; por ejemplo, compararon sus respuestas con las de sus compañeros, con lo cual mejoraron su explicación, como se evidencia en las siguientes declaraciones:

E 1: Sí, sí... estaba perdido, tiene toda la razón... ¡ Eh! Entre más grave el sonido más se agitaba la llama de la vela, como que más ondas hacían que se agitara... y no entre más agudo...

E4: "Llega un momento en que ya no es el mismo sonido fuerte, sino que es más... como más... diferente... sí... el volumen como que no tiene que ver...

Observamos que algunas veces se quedan cortos para dar sus argumentos porque no encuentran las palabras adecuadas y sus compañeros les ayudaron a complementar las ideas que iban construyendo, lo cual es un indicador de construcción colectiva de conocimiento científico.

En este caso, cuando los estudiantes buscan una explicación para lo que observan en el fenómeno, se puede asumir como un ejercicio de interpretación, pues conectan el comportamiento de la vela con el comportamiento de la membrana a través del concepto de onda o vibración. Los estudiantes presentaron diversas formas de imaginarse esas vibraciones y sus efectos, pero en el debate se fueron afinando algunas ideas. Concluyeron que había vibraciones producidas por el sonido emitido por el parlante, pero esas vibraciones eran más cortas o más largas, dependiendo de si el sonido era agudo o grave, respectivamente. 


\section{Estimulando la habilidad de inferencia}

Del anterior ciclo concluimos que este tipo de experiencias propician la participación, por tanto, se propone continuar en la misma línea, esta vez con la intensión de orientarlos a sacar conclusiones y a estimular aún más el diálogo en la clase.

\section{Experimentación para consolidar conceptos}

Objetivo: determinar la relación entre tono y frecuencia, con el fin de crear condiciones para observar la vibración del aire.

Metodología de interacción en el aula: trabajo experimental en grupos, posteriormente, trabajo individual de análisis e interpretación, para finalizar con un ejercicio de inferencia a partir de las situaciones relatadas por sus compañeros.

Actividad: construcción de flautas con pitillos, para lo cual debían determinar cómo varía el tono del sonido emitido por la flauta cuando se cambia la longitud del pitillo y cuando se hacen agujeros al pitillo (figura 3).
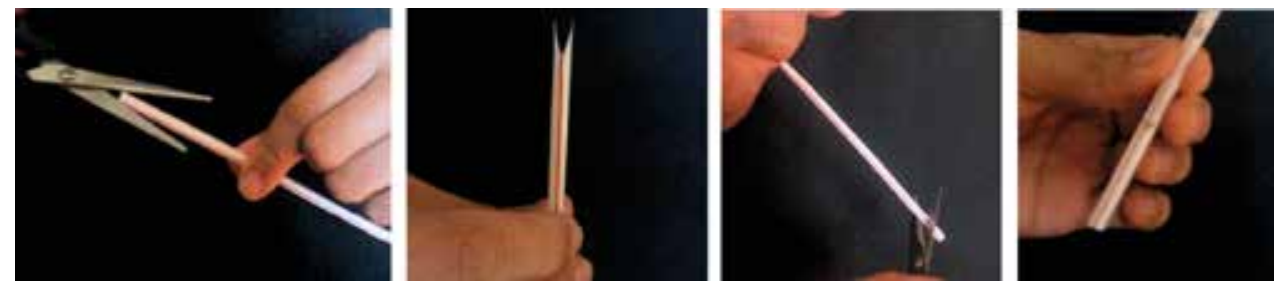

Figura 3. Proceso de fabricación de flautas utilizando pitillos

Fuente: Castiblanco, Vizcaíno e lachel, (2010)

En este caso se observó que los estudiantes encontraron evidencias de cómo se produce el sonido. Frente al pitillo, inicialmente no comprendieron cómo podría sonar y además producir diferentes sonidos; sin embargo, en el proceso de construcción lanzaron hipótesis acerca de que es necesario que pase aire por en medio de las lengüetas (puntas) hechas en el pitillo para que vibren. Cuando lo lograron, empezaron a explicar cómo lo hicieron:

G 1: Primero lo recortamos de esta forma y después solo lo soplamos y le hacíamos presión con los labios y ya.

G2: Y que pase aire, pero no mucho.

G3: Ya sonó... escuche profesora.

Posteriormente, modificamos las condiciones recortando el pitillo y haciéndolo sonar nuevamente a cada vez, o haciéndole agujeros y soplando de nuevo con algunos agujeros tapados o destapados. Allí, identificaron el concepto de tono en sonidos más agudos o más graves como una cualidad del sonido.

E4: El agudo... el que grita... el fastidioso, el pitico fastidioso... Es... ¿̇mayor frecuencia?

E5: El grave es como una trompeta.

E6: Uno es agudo y el otro es grave, ¿̇no es así? 
E7: El largo... dura bastante y es como... suspensivo por decirlo así, el otro es agudo... El largo tiende como a ser más fuerte...

Pasado un tiempo sacaron conclusiones acerca del significado del tono del sonido, así:

E8: Entre más cortico más agudo... Entre más largo más grave...

E9: Cuando está más largo, es un sonido grave y cuando esta pequeño, es un sonido agudo.

A partir de los resultados obtenidos hasta el momento pudimos ver que la estrategia utilizada creó un ambiente en que los estudiantes participaron de lo ocurrido en el aula de clase. Fue importante que vieran reflejadas sus ideas acerca de cómo se debía llevar la clase para el desarrollo del pensamiento crítico reflexivo. Sus aportes permiten evidenciar que "ese saber algo de las cosas" y "aprender en comunión con los demás", como propone Freire (2004), es posible si el profesor es consciente de su papel como agente de transformación social y se dispone a facilitar nuevos modos de interacción en la clase.

Al mismo tiempo, la docente titular empezó a construir sentido sobre la importancia de asumir la enseñanza como un proceso de investigación en el aula, en donde el diseño de las actividades estuviese inspirado en un contenido de la física, sin perder de vista que el objetivo de su enseñanza va más allá del mero contenido científico hacia el empoderamiento de los estudiantes como responsables de la construcción de su propio conocimiento; esto los hizo sentir inteligentes y capaces de participar de la clase con aportes considerados importantes por la profesora. A su vez, esta fue encontrando maneras de dialogar con sus estudiantes en torno a sus explicaciones, aun cuando las palabras no fueran exactas para describir el fenómeno.

De acuerdo con Plantin (2014), valorar los procesos de participación "es una herramienta de aprendizaje y un punto de partida de toda elaboración de una discusión" (p. 16). Esto permite que se facilite la formulación de explicaciones y argumentaciones por parte de los estudiantes ya que se sienten reconocidos como pares, lo cual es muy diferente a otorgarle todo el poder de explicación al docente. Notamos que el estímulo a la habilidad de pensamiento para la inferencia apareció cuando ellos pudieron manipular el montaje y hacer previsiones propias.

\section{Conclusiones}

Esta manera de desarrollar la clase de física, con el objetivo de propiciar el desarrollo del pensamiento crítico reflexivo, favoreció que los estudiantes conectaran sus vivencias con conceptos que explican el fenómeno del sonido. A su vez, le permitió a la docente reconocerse y encontrar un camino alternativo para la enseñanza de la física, diferente a su práctica tradicional.

Los estudiantes contrastaron sus ideas con las de los demás, hicieron preguntas como resultado de la necesidad de organizar sus propios argumentos para describir y explicar el fenómeno físico. Esto les permitió hablar asumiendo las equivocaciones, dudas o ignorancias como algo natural al analizar, ya que 
no lo veían como algo malo, sino como una posibilidad de discutir con sus compañeros hasta construir explicaciones conjuntas que les resultaran más aceptables.

La docente titular se convirtió en una facilitadora del debate que propició ambientes de aprendizaje y orientó las explicaciones de los estudiantes propiciando el diálogo entre pares. Por su parte, los estudiantes asumieron que sus aportes contribuyeron al desarrollo de la clase y permitieron la participación de todos, con el fin de expresar sus puntos de vista y opiniones.

Frente a la autorregulación, los estudiantes inicialmente presentaron dificultades para desarrollar las actividades, se dispersaban con facilidad, requerían de estar informándoles constantemente lo que debían realizar. El hecho de cambiar la manera de impartir la clase fue para ellos algo raro, pero pronto reconocieron la riqueza de exponer sus ideas frente a los demás.

De esta manera, la apuesta por presentar la clase de ciencias para la vida y de relacionar los conceptos de la física con situaciones cercanas al estudiante muestra que es posible cambiar roles. El estudiante puede sentirse protagonista, sus ideas y opiniones son tenidas en cuenta no meramente como un diagnóstico de preconcepciones sino como parte necesaria para la construcción de conocimiento.

Todo lo anterior lo entendemos como una manera de empoderar a la docente y sus estudiantes para ejercer la crítica reflexiva sobre el modo como se desarrolla la clase y, a la vez, sobre cómo se construye el conocimiento científico.

\section{Agradecimientos}

Este trabajo es desarrollado en el marco del proyecto de investigación titulado "Relaciones entre los resultados de investigación en enseñanza de las ciencias y las prácticas docentes", financiado por el Instituto de Estudios e Investigaciones Educativas de la Universidad Distrital Francisco José de Caldas.

\section{Referencias}

Castiblanco, O. y Nardi, R. (2017). What and how to teach didactics of physics? An approach from disciplinary, sociocultural, and interactional dimensions. Journal of Science Education, 19111, 100-117.

Castiblanco, O. y Vizcaíno, D. (2006). Pensamiento crítico y reflexivo desde la enseñanza de la física. Revista Colombiana de Física, 38(2), 674-677.

Castiblanco, O., Vizcaíno, D. e lachel, G. (2010). Proposta didática para o ensino do som. En II Simposio Nacional de Ensino de Ciencia e Tecnología. Brasil: Universidade Federal de Ponta Grossa.

Elliot, J. (1991). Action research for educational change. London: O.U. Keynes. Ed.

Ennis, R.H. (2013). Critical Thinking Across the Curriculum: The Wisdom CTAC program. Inquiry: Critical Thinking Across the Disciplines, 28(2), 25-45.

Facione, P. (1992). Critical thinking: what it is and why it counts. Millbrae, California CA: California Academic Press.

Flick, U. (2009). An introduction to qualitative research. Londres: SAGE Publications Ltd.

Freire, P. (2004). La pedagogía de la autonomía. Sao Paulo: Ed. Paz e Terra.

Gardner, H. (2004). Changing Minds: the art and science of changing our own and other people's minds. Vol. 1. Boston, Massachusetts: Harvard Bussines School Press.

Giroux, H. (1988). Teachers as intellectuals. Westport: Greenwood Publishing Group.

León, F. (2014). About the reflective thought also known as the critical thinking. Propósitos $y$ Representaciones, 2(1), 161-214.

Nardi, R. y Castiblanco, O. (2014). Didática da Física. Vol. 1. Sao Paulo, Brasil: Cultura Academica.

Plantin, C. (2014). Lengua, argumentación y aprendizajes escolares. Tecné, Episteme y Didaxis: TED, 36, 95-114. 
Saiz, C. (2002). Enseñar o aprender a pensar. Escritos de Psicología, 6, 53-72.

Sanmartí, N. (2002). Didáctica de las ciencias en la educación secundaria obligatoria. 1a. ed. Madrid: S. Educación, Ed.

Torres, Y. y Solbes, J. (enero de 2014). Aspectos convergentes del pensamiento crítico y las cuestiones socio científicas. Góndola, Enseñanza y Aprendizaje de las Ciencias, 911), 54-61.

\section{Para citar este artículo}

Fonseca, Y. y Castiblanco, O. (2020). Desarrollo del pensamiento crítico y reflexivo a partir de la enseñanza del sonido. Tecné, Episteme y Didaxis: TED, (47), 11 1-126. https://doi.org/10.17227/ted.num47-784 1 